\title{
The Maintenance of Genetic Variation Due to Asymmetric Gene Flow in Dendritic Metapopulations
}

\author{
Michael B. Morrissey ${ }^{1, *}$, and Derrick T. de Kerckhove ${ }^{2, *}$ \\ 1. Institute of Evolutionary Biology, School of Biological Sciences, University of Edinburgh, Ashworth Laboratories, King's Buildings, \\ Mayfield Road, Edinburgh EH9 3JR, Scotland, United Kingdom; 2. Department of Ecology and Evolutionary Biology, University of \\ Toronto, 25 Willcocks Street, Toronto, Ontario M5S 3B2, Canada
}

Submitted May 20, 2009; Accepted August 6, 2009; Electronically published October 27, 2009

Online enhancement: appendix.

ABSTRACT: Dendritic landscapes can have ecological properties that differ importantly from simpler spatial arrangements of habitats. Most dendritic landscapes are structured by elevation, and therefore, migration is likely to be directionally biased. While the populationgenetic consequences of both dendritic landscape arrangements and asymmetric migration have begun to be studied, these processes have not been considered together. Simple conceptual models predict that if migration into branch (headwater) populations is limited, such populations can act as reservoirs for potentially unique alleles. As a consequence of the fact that dendritic landscapes have, by definition, more branches than internal habitat patches, this process may lead to the maintenance of higher overall genetic diversities in metapopulations inhabiting dendritic networks where migration is directionally biased. Here we begin to address the generality of these simple predictions using genetic models and a review of empirical literature. We show, for a range of demographic parameters, that dendritic systems with asymmetric migration can maintain levels of genetic variation that are very different, sometimes very elevated, compared with more classical models of geographical population structure. Furthermore, predicted patterns of genetic variation within metapopulations - that is, stepwise increases in genetic diversity at nodesdo occur in some empirical data.

Keywords: asymmetric, migration, dendritic, metapopulations, genetic variation.

\section{Introduction}

In both spatial ecology and population genetics, the migration of individuals between populations is often represented as movement within a one-, two-, or threedimensional lattice or network of populations (Maruyama 1969; Blanchet et al. 2008). The lattice structure provides a good representation of natural systems (e.g., linear edge habitat, topological area of land, or ocean volumes), as

* Both authors contributed equally to this work.

† Corresponding author; e-mail: michael.morrissey@ed.ac.uk.

Am. Nat. 2009. Vol. 174, pp. 875-889. (C) 2009 by The University of Chicago. 0003-0147/2009/17406-51292\$15.00. All rights reserved.

DOI: $10.1086 / 648311$ well as a framework for classic population genetic models such as the linear stepping-stone (Kimura and Weiss 1964), island (MacArthur and Wilson 1967), and infinite-islands (Wright 1931) models. Recently, ecologists and geneticists have begun to explore the influence of a dendritic representation of nature on population genetic structure, community dynamics, and species distribution (Fagan 2002; May 2006; Campbell Grant et al. 2007; Labonne et al. 2008; Rodriguez-Iturbe et al. 2009). While studies have shown that dendritic arrangements of populations have ecological properties that are not found in lattice models, they have not yet incorporated influences of asymmetric movement of individuals on the genetic structure of dendritic metapopulations. Asymmetric migration in dendritic landscapes should be expected to influence the genetics of metapopulations in ways that are qualitatively different from other landscape arrangements.

A dendritic environment can be represented by a geometric pattern of arborescent bifurcation originating from one node and extending out in one direction, forming a hierarchical network of nodes and branches. The branches typically represent the route of migration between populations at nodes; however, they can also represent populations and habitats (Campbell Grant et al. 2007). Dendritic environments are very common and are found in the arrangement of populations within watersheds (Fagan 2002; Benda et al. 2004), mountain ranges (Finn et al. 2006), cave systems (Fong and Culver 1994), edge habitats (Campbell Grant et al. 2007), and, at smaller scales, on branching plants. Many of the organisms that inhabit these environments are influenced by the structure of the landscape within which they are forced to move. The arrangement of dendritic networks can influence many aspects of metapopulation ecology, including source and sink dynamics (Fagan 2002; Lowe 2002), genetic relatedness (Labonne et al. 2008), predator-prey interactions (Cuddington and Yodzis 2002), resistance to invasion (Pulliam 1988), and the distribution of local productivity (Lowe 
and Bolger 2002; Campbell Grant et al. 2007). In addition, the linear nature of migration removes the independence of population exchange often found in lattice networks, because individuals are forced to travel and interact with potentially multiple nodes to reach a location (Labonne et al. 2008). Recently, dendricity has been shown to also influence patterns of community diversity within the metacommunity framework (Rodriguez-Iturbe et al. 2009).

Dendritic habitats are often associated with changes in elevation, which in fact are generally responsible for the hierarchical spatial arrangement of these habitats. As a result of changes in elevation, movement between populations can be biased down the network due to the influence of gravity. Therefore, in general, populations may exchange individuals in an asymmetric fashion. While neither migration rate asymmetry nor landscape dendricity has been ignored in population genetics (Maruyama 1969; Lundy and Possingham 1998; Vuilleumier and Possingham 2006; Blanchet et al. 2008), both are topics of ongoing research, and the joint effects of these factors have not been explored. A general conclusion made in modeling exercises is that, all else being equal, alleles from populations with more emigrants than immigrants have a greater probability of becoming fixed for the entire metapopulation (Lundy and Possingham 1998). Asymmetric migration between populations occurs often in watersheds (Shaw et al. 1994; Schaefer 2001; Fraser et al. 2004; Markwith and Scanlon 2007), and it has also been demonstrated in mountains (Toju 2008).

The combined influence of a dendritic landscape structure and asymmetric migration is likely to have a strong influence on the genetic structure of a metapopulation (Labonne et al. 2008) and contribute to a significant departure from outcomes in classic population genetic models (Donnelly and Tavaré 1995). Take the simple illustrative case of a watershed with unidirectional gene flow such that two isolated headwater populations provide migrants to one downstream population. This type of gene flow is found in many fluvial systems and typically is created by the flow of water over barriers such as waterfalls (Shaw et al. 1994; Deiner et al. 2007) and the passive drift of gametes, larvae or seeds, and adults of many organisms (e.g., Bilton et al. 2001; Markwith and Scanlon 2007). In the absence of mutation, the equilibrium level of diversity in such a system is nonzero, with a probability corresponding to the initial number of alleles and their initial frequencies. For example, if the system initially contains two alleles segregating at equal frequency, the headwaters will become fixed, due to drift for alternate alleles, at a probability of 0.5 . When this occurs, variation will be maintained indefinitely. As the number of headwater populations in the dendritic structure increases, so too does the probability of maintaining a genetic polymorphism (e.g., 0.996, i.e., $1-0.5^{9-1}$ for nine headwater populations, as in fig. $1 B$ ). Under these conditions, maintenance of polymorphism in the metapopulation as a whole is nearly guaranteed (Hanski 1999); thus, we expect asymmetric gene flow in dendritic landscapes to promote the maintenance of polymorphism, particularly in main-stem populations (Hanski 1999; Vuilleumier and Possingham 2006). This elementary outcome is unique among population genetic models with drift and no mutation, where other population-genetic models predict diversities of 0 at equilibrium. These predictions can only be achieved by intuition for models that do not allow mutation, selection, or varying degrees of asymmetric migration.

In order to investigate the potential for asymmetric migration in dendritic landscapes to promote the maintenance of genetic variation, we use deterministic models and individual-based simulations to further the simplified watershed model introduced above. We contrast the population genetics of 10 populations arranged in three spatial structures that are likely to be found in aquatic environments: a dendritic structure (fig. $1 A$ ), a uninodal structure with multiple headwaters feeding into one main stem (fig. $1 B$ ), and a linear stepping-stone structure (fig. 1C). The deterministic models allow us to use standard approaches from population genetics to explore the influence of asymmetry and dendricity on the maintenance of genetic variation over a wide range of demographic parameters. Individual-based simulations allow conceptually simple models to be constructed of natural phenomena, easing interpretation and providing validation for more complex

\section{(A)}

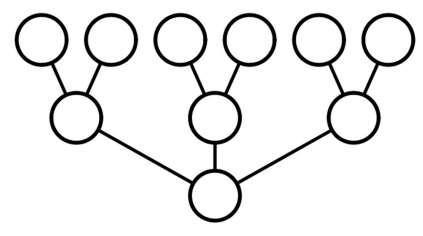

(B)
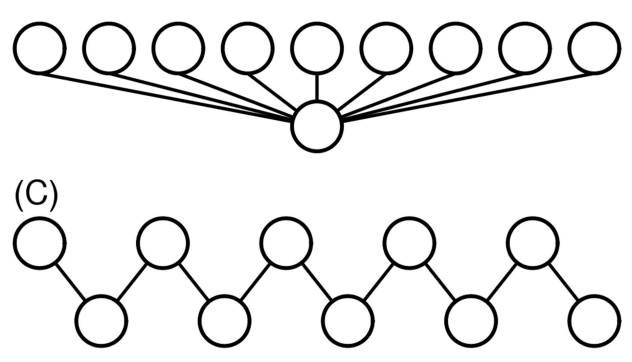

Figure 1: Landscape arrangements used to test for effects of landscape dendricity and asymmetric migration on the maintenance of variation in metapopulations. The landscape arrangements are referred to in the text as dendritic $(A)$, uninodal $(B)$, and linear stepping-stone $(C)$. 
approaches. We recognize that these same interactions may occur in nonaquatic environments, but for the sake of clarity and a consistency in terms, we will discuss our models primarily in the context and terminology of watersheds.

\section{Analytical Approaches}

Probabilities of identity by descent for any two genes in a metapopulation can be described by the recursive equation

$$
1-F_{i j, t+1}=\sum_{i j, k l} G_{i j, k l}\left(1-F_{k l, t}\right),
$$

where $F_{i, j}$ is the probability that an allele sampled from deme $i$ at time $t$ is identical by descent to an allele sampled at $t$ from deme $j$ (Whitlock and Barton 1997). In this equation, $G_{i j, k l}$ is the probability that an allele from deme $i$ is descended from an allele in deme $k$ in the previous generation and that an allele from deme $j$ is descended from a distinct allele in deme $l$. Therefore, $\mathrm{G}$ is an $n^{2} \times n^{2}$ matrix where $n$ is the number of demes and with elements that are easily calculated from migration rates between demes and population sizes of the demes, assuming that these demographic parameters are fixed. Elements of $\mathbf{G}$ can be obtained for any arbitrary migration matrix from the product $m_{i k} m_{j l}$. For example, the matrix $\mathbf{m}$ for the uninodal arrangement of populations in figure $1 B$ is

$$
\mathbf{m}_{\text {uninodal }}=\left[\begin{array}{lllll}
a & d & d & d & \cdots \\
u & b & 0 & 0 & \\
u & 0 & b & 0 & \\
u & 0 & 0 & b & \\
\vdots & & & & \ddots
\end{array}\right],
$$

where $u$ is the upstream migration rate, $d$ is the downstream migration rate $a=1-9 u$, and $b=1-d$. This full matrix and the matrices for the other two landscape arrangements in figure 1 are given in the appendix in the online edition of the American Naturalist. Note that this example is a forward-migration matrix, in the sense that it specifies individual probabilities of migrating, whereas equation (1) requires retrospective migration rates, that is, probabilities that individuals have migrated. These are not the same when migration is not conservative, that is, when populations do not have equal numbers of immigrants and emigrants; see the appendix for calculations of the appropriate migration rates.

The leading nonunit eigenvalue of $\mathbf{G}$ gives a measure of the effective population size $\left(N_{e}\right.$; this is the eigenvalue for effective population size; see Ewens [1982] for a discussion of its relationship to other measures of effective population size). The $N_{e}$ of a metapopulation can be thought of as the size of a hypothetical Fisher-Wright population that would behave in a similar manner with respect to the dynamics of changes in probabilities of identity by descent. Patterns in the ratio of $N_{\mathrm{e}}$ to census size $(N)$ for any pattern of connectivity among demes, migration rates among demes, and deme sizes provides a useful indicator of the effect of varying demographic conditions on the force of drift in a metapopulation (Palstra and Ruzzante 2008). Figure $2 A-2 C$ shows $N_{\mathrm{e}}: N$ ratios for all three patterns of connectivity (fig. $1 A-1 C$ ), for mean migration rates varying from $10^{-5}$ to $10^{-3}$, for asymmetries in migration rate varying from unidirectional migration upstream to unidirectional migration downstream, and for $N=100$ in all demes.

Population structure and migration asymmetry are the main factors effecting variation in relative $N_{\mathrm{e}}$ for the majority of the ranges of population structure, mean migration rates, and degrees of asymmetry examined, as evidenced by the predominantly horizontal patterning of the contour lines in figure $2 A-2 C$. Migration rate biased in the upstream direction results in decreases in $N_{\mathrm{e}}$ in all population structures due to gene swamping by a single source population. In contrast, downstream biases in migration rates can substantially reduce the effect of drift in the dendritic and uninodal model population structures. Even for migration rates that would effectively homogenize allele frequencies among demes in stepping-stone or island population genetics models (i.e., $N m \approx 1$ or $m=0.01$ in fig. 2), with modest degrees of asymmetry (i.e., -0.5 in fig. $2 A-2 C$ corresponds to a value three times greater in downstream migration than upstream migration), the force of drift in dendritic population structure acts at rates equivalent to those that would occur in populations more than twice as large. Upstream and downstream biases in migration rate have equivalent impacts on $N_{\mathrm{e}}: N$ ratios in the linear population structure such that, unlike in the other population structures, migration rate asymmetry uniformly promotes the loss of variation. The relative pattern between landscape arrangements is robust to changes in demographic properties, including unequal population sizes and migration rates (see the appendix).

Iteration of equation (1) provides deterministic predictions of fixation probabilities within and between demes. Figure $2 D-2 F$ shows the dynamics of all classes of $F_{i j}$ for the population structures where $i=j$, that is, fixation probabilities within demes, for asymmetric migration where $m_{\text {downstream }}=10^{-4}$ and $m_{\text {upstream }}=10^{-3}$, and for $N=100$ in all demes, from a hypothetical set of starting values where all $F_{i j}=0$. Figure $2 G-2 I$ shows the equivalent patterns in expectations of $F_{i j}$ where $i \neq j$, that is, fixation probabilities for alleles sampled from different demes but for the same population structures and migration rates. 

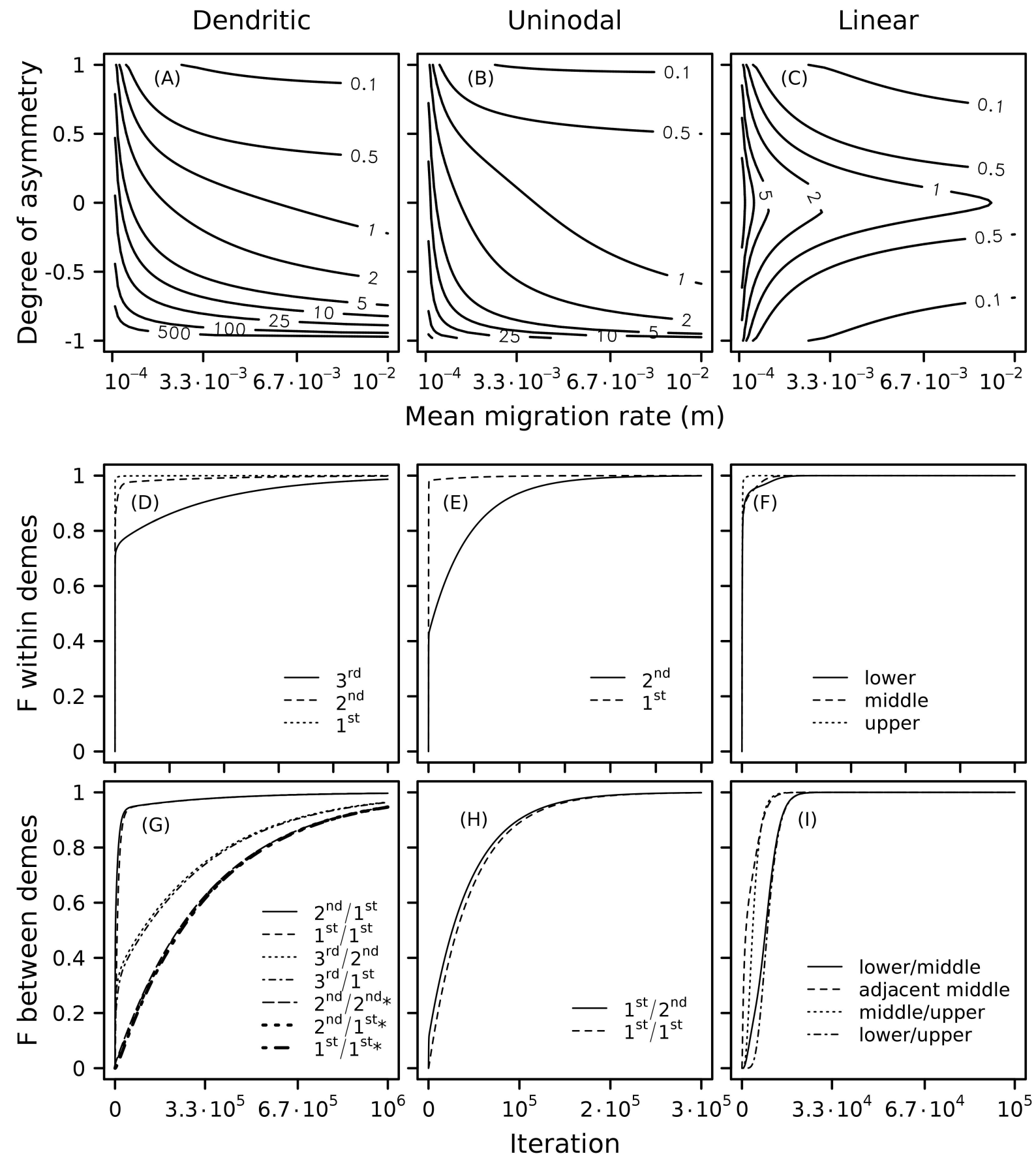

Figure 2: $A-C$, Relative effective population size ( $N_{\mathrm{e}} /$ census size) in dendritic, uninodal, and linear landscape arrangements. Upstream migration rates are $(\alpha+1) \times m$, and downstream migration rates are $|(\alpha-1) \times m|$, where $\alpha$ is the migration rate asymmetry and $m$ is the average migration rate. Thus, for example, values of $-1,0$, and +1 correspond to unidirectional downstream, unbiased, and unidirectional upstream migration, respectively. Probabilities of identity by descent are within demes in $D-F$ and between demes in $G-I$ as a function of time from a hypothetical starting point of all $F=0$ in the three landscape arrangements. Ordinals in the plot legends $(1 s t, 2 n d, 3 r d)$, refer to stream order, where first-order streams are headwaters, second-order streams are fed by at least two first-order streams, et cetera; pairs of stream orders marked with an asterisk indicate $F_{i j}$ values for demes in different subcatchments; that is, they are connected via the third-order stream. Deme sizes are 100 in all scenarios, and 10 demes compose each metapopulation. In $D-I$, upstream migration rates of $10^{-4}$ and downstream migration rates of $10^{-3}$ were applied. See figure 1 for specific arrangements of connectivity between demes. In $G$, the entries in the legend are arranged in the order of the highest to lowest curves in the plot. 
These migration rates represent substantial but not absolute downstream bias in migration, as well as a level of gene flow that maintains connectivity while allowing population differentiation to evolve. Note the differences in temporal scale among figure $2 D-2 I$.

Figure 2 illustrates the mechanism through which migration rate asymmetry counteracts drift in dendritic landscapes. The maintenance of different alleles in disparate headwater populations (fig. $2 \mathrm{G}, 2 \mathrm{H}$ ) in our illustrative dendritic and uninodal population structures and demographic parameters can occur for vastly longer than it can in the linear structure (fig. 2I). Thus, while variation is lost very quickly within headwater populations (fig. $2 D$ $2 F$ ), the low probability that the same alleles become fixed in these different populations results in the maintenance of genetic variation.

Following Nagylaki (1998), expected coalescence times for general migration matrices and population sizes can be described by

$$
T_{i j}=1+\sum_{k l} m_{i k} m_{j l} T_{k l}-\sum_{k} \frac{m_{i k} m_{j k}}{2 N_{k}} T_{k k},
$$

where $T_{i j}$ is the expected coalescence time of a pair of distinct alleles sampled from demes $i$ and $j, m_{i j}$ is the probability that an allele sampled in $i$ is descended from an allele in $j$ in the previous generation, and $N_{i}$ is the effective population size of deme $i$. While equation (2) can be used to produce simple expressions for $T_{i j}$ and population genetic parameters, such as for the number of segregating sites under simple mutation models, derivation of simple expressions for the patterns of migration that are likely to occur in dendritic landscapes does not appear to be possible. This is partly because not all populations are equivalent and migration is not conservative; that is, some or most demes have unequal numbers of immigrants and emigrants. Nonetheless, equation (2) can be used to calculate useful population genetic parameters for dendritic systems.

Rearrangement of equation (2) yields

$$
\begin{aligned}
1 & =\sum_{k l} a_{i j, k l} T_{k l}, \\
a_{i j, k l} & =\delta_{i=k, j=l}+\gamma_{k=l} \frac{m_{i k} m_{j k}}{2 N_{k}}-m_{i k} m_{j l},
\end{aligned}
$$

where $a_{i j, k l}$ are the elements of a matrix defining the coefficients of a system of linear equations relating $T_{i j}$ to an arbitrary migration matrix, $\mathbf{m}$, and vector of deme sizes, $\mathbf{N}$. The variables $\delta_{i=k, j=l}$ and $\gamma_{k=l}$ are indicator variables that have values of 1 when the conditions of their subscripts are satisfied and values of 0 otherwise.
Specific values of $T_{i j}$, as obtained from the solution to the system of equations defined by equation (3), can provide information on the force of drift in any given metapopulation structure. Values of $T_{i j}$ when $i=j$ provide an indication of the force of drift within populations, and values of $T_{i j}$ when $i \neq j$ are an indication of subdivision between populations $i$ and $j$. The effect of drift acting on the metapopulation as a whole is indicated by the (weighted) expected coalescence time $\sum_{i j} N_{i} N_{j} T_{i j} N_{\mathrm{T}}^{-2}$, where $N_{\mathrm{T}}$ is the sum of the size of all of the demes. Similarly, $F_{\mathrm{ST}}$ can be obtained as the quotient of $\sum_{i} N_{i} T_{i i} N_{\mathrm{T}}^{-1}$ and the mean coalescence time for a pair of alleles (Charlesworth et al. 2003).

Figure $3 A-3 C$ shows expected coalescence times derived from equation (3) for dendritic, uninodal, and linear landscapes, each with 10 demes and local population sizes that range from $N=10$ to $N=10^{4}$ diploid individuals. Corresponding $F_{\mathrm{ST}}$ values are invariant with respect to population size; they are 0.92 and 0.81 for the dendritic landscapes with asymmetric and symmetric migration, respectively, and 0.88 and 0.75 for the uninodal landscapes with asymmetric and symmetric migration, respectively. As for expected coalescence times, the pattern in $F_{\mathrm{ST}}$ is reversed with respect to migration rate asymmetry in the linear landscape; values are 0.82 and 0.87 with asymmetric and symmetric migration, respectively.

Because of a lack of analytical solutions for distributions of coalescence times for general migration matrices and population sizes, observable genetic properties of metapopulations such as heterozygosity cannot be derived. However, coalescent simulations provide computationally efficient means of obtaining expectations of population genetic parameters in subdivided populations (Rosenberg and Nordborg 2002). For general patterns of migration and finite numbers of demes, distributions of coalescent times can be approximated by simulating genealogies of pairs of gene copies. From such simulations, patterns of genetic diversity can be obtained by simulating mutation models on top of the simulated genealogies. Figure $3 D-$ $3 I$ shows expected heterozygosities in each of the model population structures depicted in figure 1 , as obtained from coalescent simulations under an infinite-alleles model with a mutation rate of $10^{-5}$ and from a stepwise mutation model with a mutation rate of $10^{-3}$, for a range of deme sizes from 10 to $10^{4}$. These mutation models and rates roughly mimic gene and microsatellite loci, respectively. In these simulations, total upstream migration rates (i.e., $\mathrm{Nm}$ ) were held constant at 0.01 and total downstream migration rates were held constant at 0.1 . Thus, variation in $H_{\mathrm{e}}$ in these simulations will result from variation in population size and landscape arrangements, rather than from changes in the number of migrants corresponding to variation in population size. 

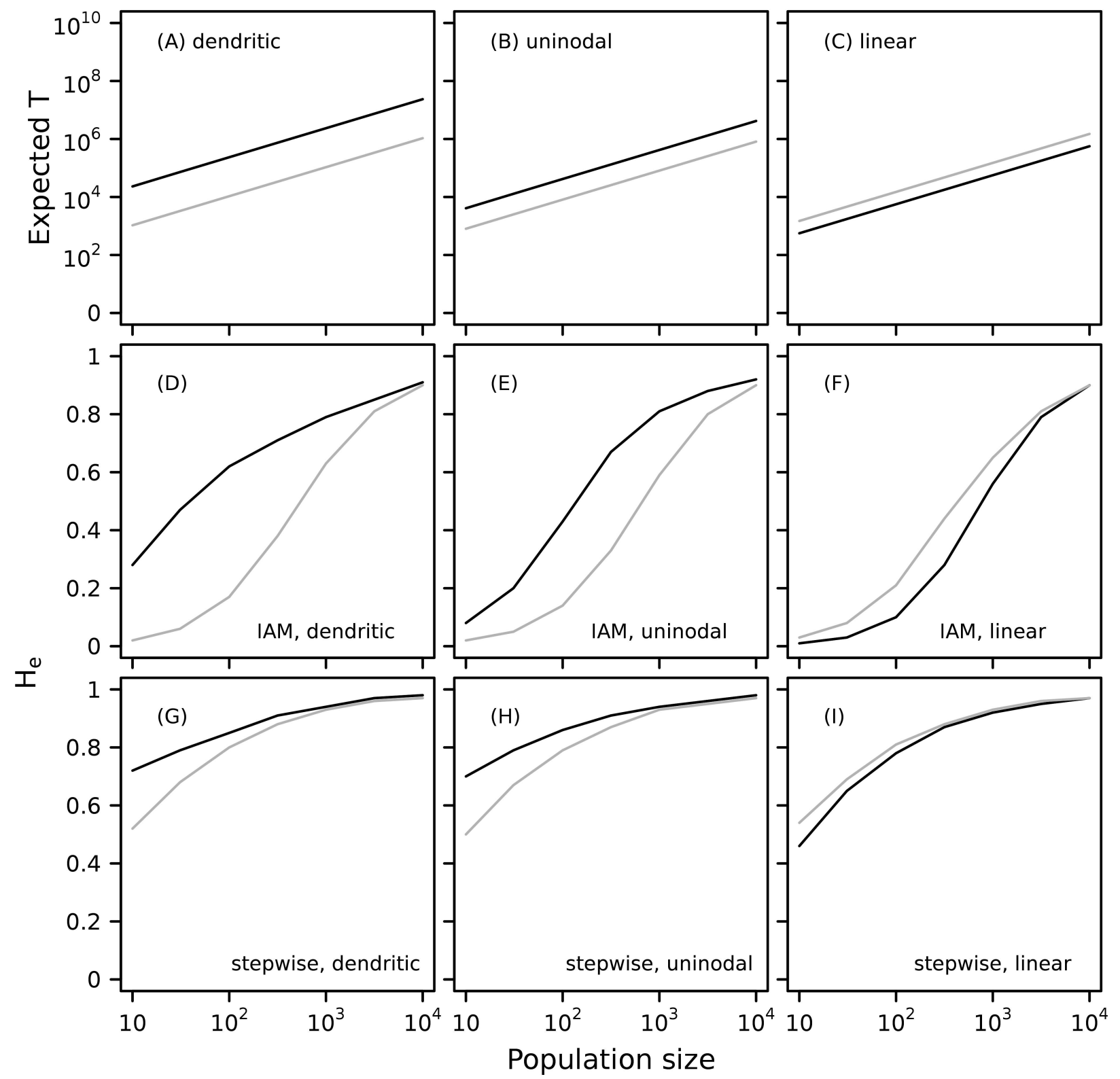

Figure 3: Expected coalescence time $(T ; A-C)$ and heterozygosity $\left(H_{\mathrm{e}} ; D-I\right)$ in metapopulations composed of demes ranging in size from 10 to $10^{4}$ individuals. In $D-F$, an infinite-alleles mutation model was applied with a mutation rate of $10^{-5}$, and in $G-I$, a stepwise mutation model with a mutation rate of $10^{-3}$ was applied. Black lines denote $T$ and $H_{\mathrm{e}}$ under asymmetric migration, with upstream and downstream migration rates of $10^{-4}$ and $10^{-3}$, respectively. Gray lines denote $T$ and $H_{\mathrm{e}}$ under symmetric migration, with migration rates in both directions of $5.5 \times 10^{-4}$. See figure 1 for specific arrangements of connectivity between demes.

These coalescent simulations show that the combined effects of migration rate asymmetry and dendritic landscape arrangements to promote the maintenance of genetic variation can be expected to be manifested in patterns of variation at real loci over a range of population sizes. However, the equilibrium patterns in diversity vary with the type of locus. For the model mimicking the mutation of a gene locus, such as what might be assessed via single nucleotide polymorphisms or allozymes, a substantially higher $H_{\mathrm{e}}$ value is maintained in the dendritic and uninodal model population structures under downstreambiased asymmetric migration for population sizes from about $10^{2}$ to $10^{3}$. This pattern in $H_{\mathrm{e}}$ is not expected to be as strong for microsatellite loci. We should note, however, 
that at highly polymorphic loci, large differences in levels of polymorphism can occur with small corresponding differences in heterozygosity (Chen et al. 2009). The small differences in $H_{\mathrm{e}}$ at the microsatellite loci as predicted by figure 3 in fact correspond to substantial differences in the number of alleles maintained in the metapopulations. For example, for a population size of 1,000 in the dendritic landscape structure, expected heterozygosities of 0.79 and 0.63 are maintained under asymmetric and symmetric migration, respectively. This numerically small difference in $H_{\mathrm{e}}$ corresponds to 4.8 and 2.7 effective alleles, respectively (i.e., the number of equally frequent alleles required at a locus to generate the observed level of heterozygosity; actual numbers of alleles are typically higher because of allele frequency skew), and thus these patterns in $H_{\mathrm{e}}$ may correspond to meaningful and detectable differences in genetic diversity in nature at microsatellite loci as well.

\section{Individual-Based Simulation Models}

The deterministic models that we have considered so far suggest that variation can be maintained for much longer periods in dendritic and uninodal metapopulation arrangements with asymmetric gene flow than in linear systems or under symmetric migration. To further explore how diversity is maintained under nonequilibrium conditions and among loci, we modeled metapopulations in the landscape arrangements in figure 1 using individualbased simulations. Individual-based simulations offer intuitive and flexible representations of natural systems by tracking individuals and the spatial environment over many generations (DeAngelis and Mooij 2005). They also offer an opportunity to compare results from deterministic models with results from a conceptually simpler approach (DeAngelis et al. 2007).

Each simulation consisted of 1,000 diploid individuals reproducing sexually in nonoverlapping generations and divided into 10 populations arranged in one of the three landscapes depicted in figure 1. For the first generation of any simulation, all individuals were assigned a sex (assuming a balanced sex ratio) and the same homozygotic genotype for multiple loci (for simulation scenarios with initially polymorphic loci, see appendix). In subsequent generations, each individual genotype was constituted from the genotypes of randomly chosen parents of each sex, assuming Mendelian inheritance. We incorporated a general migration matrix where any individual has an arbitrary probability of migrating to any population in the simulated metapopulations. Specifically, we implemented the same migration matrices and average migration rates used to simulate symmetric and asymmetric migration in the deterministic models. All simulation scenarios were run for $10^{5}$ generations in order to provide short-term, potentially nonequilibrium predictions, as well as estimates of equilibrium expectations and patterns.

Loci in the simulation had the ability to undergo mutation. We simulated a $k$-alleles mutation model with $k=100$ possible types and a mutation rate of $10^{-5}$. In this $k$-alleles model, mutations occurred to any of the $k$ states with equal probability, that is, independently of allelic state and including silent mutations at a rate of $1 / k$. As such, this $k$-alleles model was intended to mimic evolution at a gene locus with a moderate number of neutral or nearly neutral alternate states that can be achieved by mutation on modest timescales. This $k$-alleles mutation model is more easily implemented in an individual-based model than in the infinite-alleles model, but it is expected to very closely approximate the infinite-alleles model for $1 /\left(1-H_{\mathrm{e}}\right) \ll k$, as is the case in all scenarios here (Hamilton 2009). Furthermore, nearly identical results are obtained under both mutation models in coalescent simulations for a wide range of parameters, including those simulated here.

Equilibrium or long-term predictions of patterns of variation in heterozygosity among the different population structures based on the individual-based model (fig. 4) are very similar to predictions based on coalescent simulations (fig. 3) and inferences of the efficacy of drift based on iteration of the deterministic model (fig. $2 D-2 F$ ). However, the values of heterozygosity that were ultimately obtained were not reached until approximately $2 \times 10^{4}$ generations. This very slow approach to the equilibrium expectations is not surprising, given the long times required for identity by descent probabilities to reach unity (fig. $2 E-2 I$ ) and the relatively low mutation rate that we simulated. Notably, equilibria are approached faster for faster-evolving loci such as microsatellites (see appendix). Despite the slow approach to equilibrium values, the ultimate pattern of differences in heterozygosity among populations at different landscape positions was achieved very quickly, where greater genetic diversity evolved in mainstem populations in the dendritic and uninodal population structures in fewer than $10^{3}$ generations. Furthermore, the time required for equilibrium patterns to emerge is strongly dependent on the initial levels of diversity; equilibrium-like patterns of diversity evolve very quickly when loci are initially variable (appendix). An important feature of the dendritic system is that genetic variation increases in a stepwise manner at nodes. This occurs to only a very small extent when migration is symmetric, reflecting the fact that the landscape arrangement itself imposes some asymmetry in migration patterns. However, the effect was small relative to the interaction of individual migration rate asymmetry and landscape structure.

At equilibrium, variation in heterozygosities among populations in different stream orders was substantial rel- 

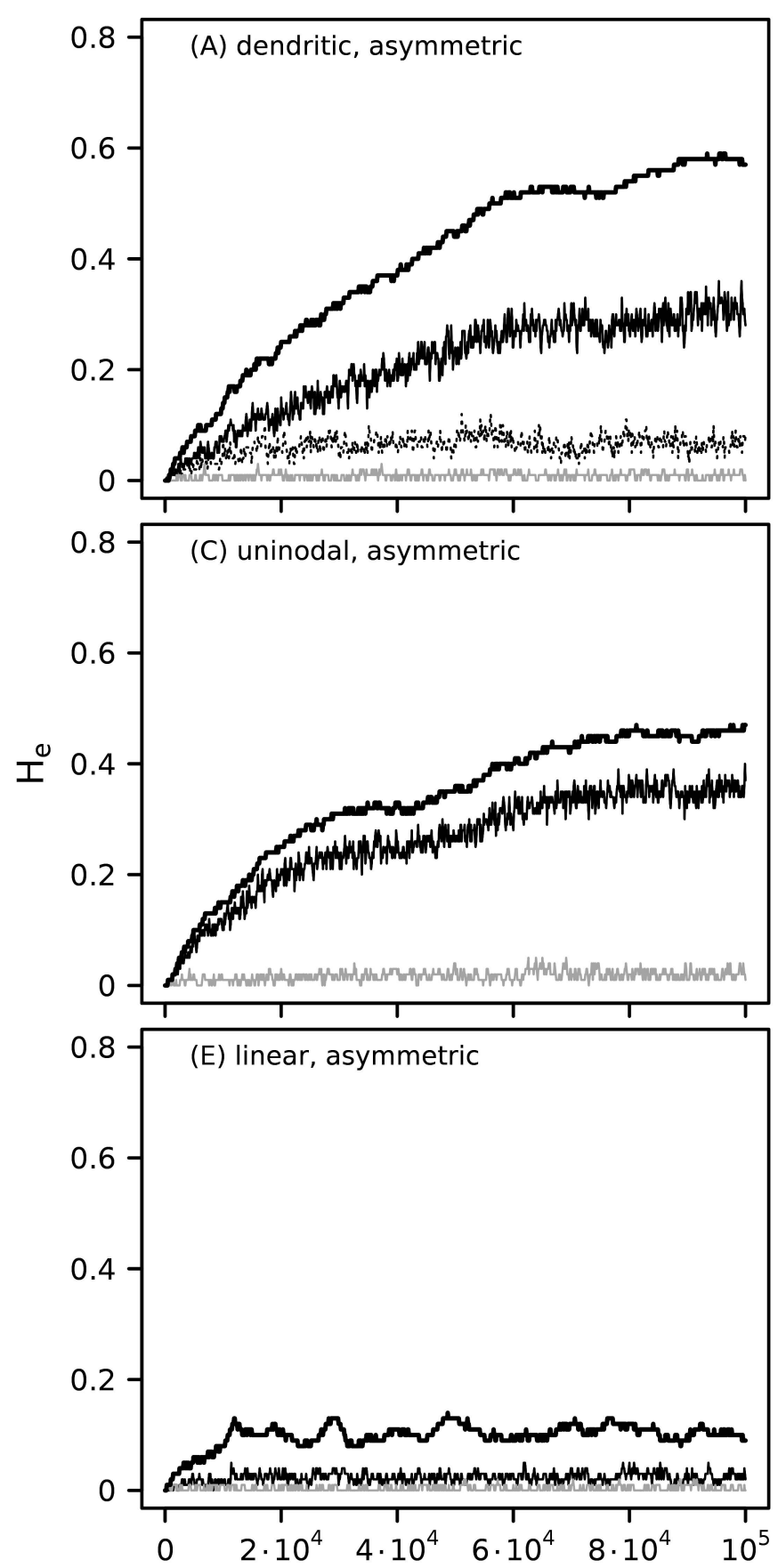

(B) dendritic, symmetric
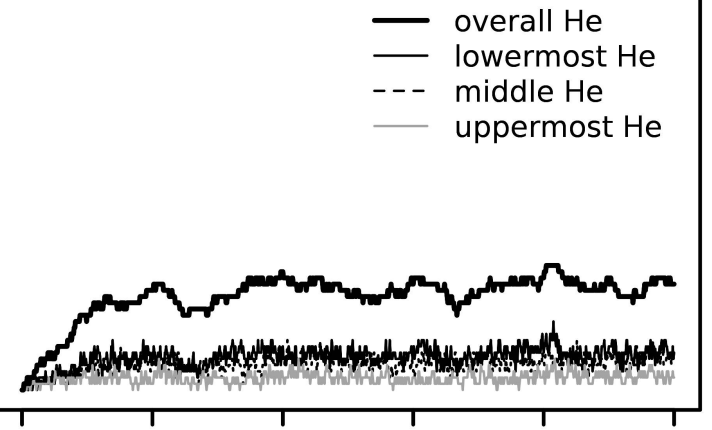

(D) uninodal, symmetric

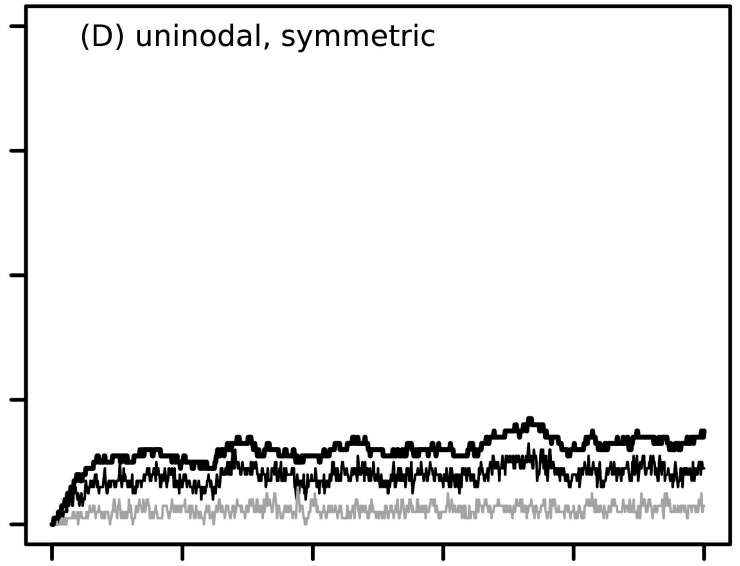

(F) linear, symmetric

Generation

Figure 4: Expected heterozygosity $\left(H_{\mathrm{e}}\right)$ in dendritic, uninodal, and linear landscape arrangements under symmetric and asymmetric migration, as predicted by individual-based simulation. A $k$-alleles mutation model was applied, with $k=100$ and a mutation rate of $10^{-5}$. Under asymmetric migration, upstream and downstream migration rates are $10^{-4}$ and $10^{-3}$, respectively. For symmetric migration, rates in both directions are $5.5 \times 10^{-4}$. All lines represent means across 10 loci from each of 10 combined simulations. See figure 1 for specific arrangements of connectivity between demes. In the key, "uppermost" refers to headwater streams, "lowermost" refers to streams of the highest order (or most downstream locations), and "middle" refers to the second-order streams in the dendritic landscape. Overall $H_{\mathrm{e}}$ refers to expected heterozygosity across populations, that is, $H_{\mathrm{T}}$. 
ative to variation among loci in the dendritic and uninodal population structures (fig. 5). These relative levels of variation within and among populations suggest that patterns of variation caused by interactions between dendritic population structures and asymmetric migration are potentially detectable at real loci. Importantly, these marked patterns in genetic variation among populations developed long before equilibrium values were reached, particularly for simulations of faster-evolving marker loci (see fig. A4 in the online edition of the American Naturalist), and thus such patterns may be detectable in landscape arrangements that have not necessarily been constant for very long periods of time.

\section{Discussion}

Our model-based results clearly demonstrate three important features of the genetics of metapopulations in dendritic landscapes. First, the dendritic arrangements of populations under downstream asymmetric migration leads to the maintenance of higher genetic diversity than standard genetic models of metapopulations do, including the linear stepping-stone (this study; Maruyama 1970) and infinite-islands (Wright 1931) models. Second, the maintenance of variation is due neither to the spatial arrangements of the populations nor to the asymmetric exchange of individuals alone, but rather to the interaction of the
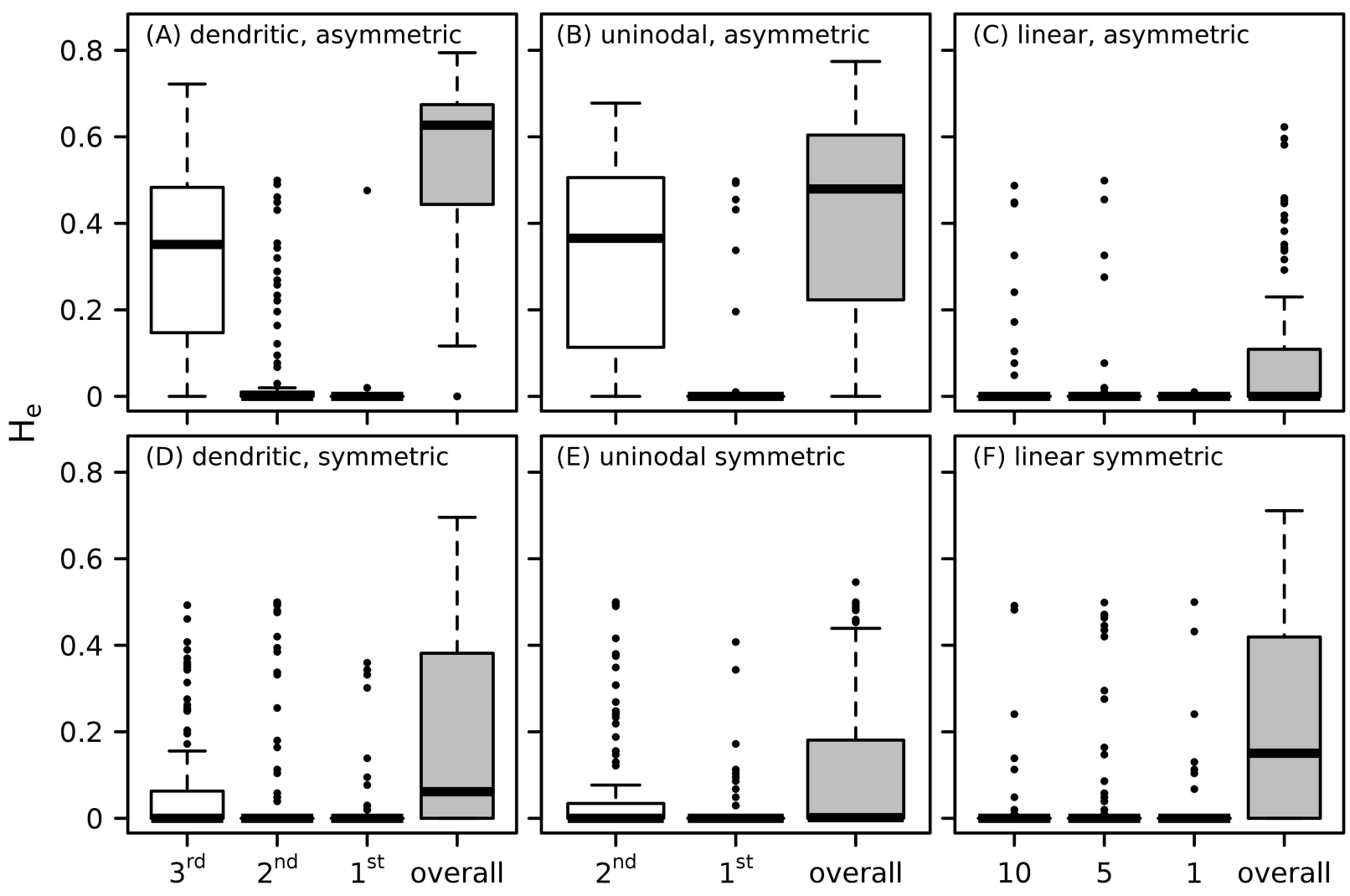

Stream order or position

Figure 5: Distributions of expected heterozygosity $\left(H_{\mathrm{e}}\right)$ among loci within populations in dendritic, uninodal, and linear landscape arrangements under symmetric and asymmetric migration, as predicted by individual-based simulation after $10^{5}$ generations. For low-order stream positions, where multiple equivalent populations exist, distributions are given for a single population. A $k$-alleles mutation model was applied, with $k=100$ and a mutation rate of $10^{-5}$. Under asymmetric migration, upstream and downstream migration rates are $10^{-4}$ and $10^{-3}$, respectively. For symmetric migration, rates in both directions are $5.5 \times 10^{-4}$. Rectangular areas of plots represent the first quartiles above and below the media, denoted by the solid black line. Stream positions for the linear landscape arrangement are 1-10 for the uppermost to lowermost populations, relative to the direction of migration in the asymmetric simulations; that is, population 1 has the net deficit of migrants. Error bars are drawn to 1.5 times the interquartile range. See figure 1 for specific arrangements of connectivity between demes. 
two. Third, the maintenance of variation does not necessarily require extreme values in asymmetric migration rates, equilibrium conditions, or long time periods following the initial colonization. Many important questions remain to be explored in both empirical and modeling frameworks, including field studies of asymmetry in dendritic systems and the incorporation of demographic variability (such as extinction-recolonization dynamics) into the models. We can nonetheless conclude that asymmetric migration in dendritic landscapes should have important effects on the genetics of metapopulations, and that these effects differ significantly from the predictions of classical genetic population models.

Under symmetric migration, there is little difference in the maintenance of genetic variation between the three landscape arrangements. It is only under asymmetry that the maintenance of variation is depressed in the linear system and elevated in the uninodal and dendritic systems. It is of considerable interest that the uninodal system is not as effective as the hierarchical arrangement of nodes and branches in the dendritic network at maintaining diversity. In our models, we find that asymmetric migration in the dendritic arrangement produces higher relative $N_{\mathrm{e}}$ values (fig. 2) and levels of heterozygosity (fig. 4) relative to the uninodal arrangement, despite having fewer headwater populations, that is, fewer reservoirs for potentially unique alleles. The tendency of an allele from any given population to become fixed is a function of its ability to colonize all demes in the metapopulation. In dendritic systems, this includes the colonization of all headwaters. Under asymmetric migration, multiple (rare) upstream migration events are required for metapopulation-wide fixation to occur, and as such each node can be considered to be a buffer against the eventual fixation of alleles. Therefore, in a minimally developed system, fewer sequential nodes exist and fixation becomes more likely. However the uninodal system is more than a special case of reduced dendricity. If a dendritic network is analogous to a watershed, then the uninodal system is analogous to a closedsystem lake with multiple tributaries. There is only one level of hierarchy in a uninodal system: that between the many headwaters and the single downstream node. The dynamics of gene flow in this system are unique, as the lack of nodal buffers results in a high sensitivity between headwater and lake genetics. This leads to the greater homogenization of allele frequencies found in our models and in studies of maladaptive gene flow in lakes with headwater tributaries (Hendry and Taylor 2004).

An interesting difference between the genetic models of dendritic metapopulations that we have presented here is that sources of genetic diversity-that is, headwater populations-are themselves genetically homogeneous. In other genetic models where populations differ in genetic diversity, populations that act as sources of diversity are themselves diverse, for example, in the continent-island model (MacArthur and Wilson 1967) or in models of species range limitation (Kirkpatrick and Barton 1997). Dendritic systems with asymmetric migration may be viewed as specific and extreme cases of population subdivision promoting the maintenance of genetic variation, which is akin to problems in the management of endangered species where breeding schemes with subdivision can maximize the maintenance of specieswide variation (Margan et al. 1998). In this case, however, many populations do not receive migrants and can therefore be particularly genetically depauperate (fig. 4). Management implications of this particular situation and positive roles for small genetically isolated populations remain to be investigated (but see Northcote 1992 for some discussion of the value of such populations).

While the mechanisms leading to the maintenance of genetic variation are different from those that maintain species diversity (although see Velland 2005), expected patterns of genetic diversity are similar to patterns in other ecological properties of dendritic systems. Higher species diversity is generally found in networks with higher numbers of nodes and branches (Campbell Grant et al. 2007). This has been attributed to the effect of nodes that promote species coexistence, and it has been found, for example, in cave colonization (see Fong and Culver 1994), invasion scenarios (see Pulliam 1988), and predator-prey interactions (Cuddington and Yodzis 2002). In addition, the use of neutral metacommunity models involving anisotropic dispersal in dendritic networks have led to strikingly similar results for community diversity to those found in our models. High directionality in dispersal maintained much higher species diversity in a simulated river network as compared with a simulated savannah (Rodriguez-Iturbe et al. 2009). As in our models, the average local species diversity was lower in the river networks, but a high level of community dissimilarity between patches led to the higher overall species diversity. In a similar neutral model, the patterns of fish species diversity in the MississippiMissouri basin in the United States were well represented using mainly dispersal, habitat capacity, and the spatial patterning of the network (Muneepeerakul et al. 2008).

The simulations mimicking a gene mutation approach equilibria very slowly, requiring at least 20,000 generations to approach long-term values. This was especially true in the scenarios that involved asymmetric migration in dendritic landscapes, where equilibria were reached after about 60,000 generations (fig. 4). This is a consequence of the very slow approach to equilibrium values of probabilities of identity by descent (fig. 2). Given the generation times of many organisms and the temporal scales during which drainage arrangements are constant, these equilibria are 
unlikely to be reached in many systems. For example, the current drainage arrangement within the Laurentian Great Lakes in eastern North America has only existed for about 6,000 years (Fulton 1989; Mandrak and Crossman 1992), and even in nonglaciated landscapes, drainages can change due to processes such as river capture or anthropogenic diversions (Bishop 1995). But, while genetic processes may reach equilibria in few watersheds on these timescales, asymmetric migration may nonetheless lead to elevated levels of diversity. After fewer than 10,000 generations, diversity was elevated in the dendritic simulations with asymmetric migration, relative to symmetric migration (fig. 4). In addition, if historical source populations are not completely homozygous, equilibria in natural systems may be reached faster. While founder effects or drift in glacial refugia may contribute to low initial diversity, genetic variation is unlikely to be entirely absent. In some cases, colonists might have relatively high diversity. For example, much of the waters of North America were colonized by a mix of lake trout (Salvelinus namaycush) from different Pleistocene glacial refugia as a result of mixing of strains in proglacial lakes (Wilson and Hebert 1998). Even in the absence of mutation (see fig. A2 in the online edition of the American Naturalist), the erosion of this initial diversity has the potential to be effectively stalled in dendritic landscapes relative to the timescales at which drainage patterns are constant in temperate regions (Fulton 1989; Mandrak and Crossman 1992).

There are few empirical examples available for verifying the patterns of genetic diversity that emerge in the theoretical models. In an extensive literature review conducted for this study, only 27 articles examined population genetics in dendritic systems where asymmetric movements likely occurred, and only seven studying fish (Yamamoto et al. 2004; Wofford et al. 2005; Crispo et al. 2006; Hanfling and Weetman 2006; Deiner et al. 2007; Raeymaeker et al. 2008; Barson et al. 2009), one studying aquatic plants (Fer and Hroudova 2008), and one studying zooplankton (Derry et al. 2009) provided appropriate systems to investigate or evaluate the results from our modeling exercises. Although there are many empirical studies of the population genetics of aquatic species in fragmented environments or exhibiting asymmetric migration (Saillant et al. 2004; Heggenes and Red 2006; Haponski et al. 2007; Reid et al. 2008; Chen et al. 2009), there are few in dendritic systems that include comprehensive sampling of headwaters, intermediate branches, and the main stem, and none that allow for comparisons of local and overall genetic diversity between similar watersheds that differ only in the capacity for dispersal or dendricity.

Nonetheless, results from a few of the available empirical studies are insightful. Hanfling and Weetman (2006), Crispo et al. (2006), and Fer and Hroudova (2008) ex- amined genetic variation in the river sculpin (Cottus gobio) in the River Rye in northeast England, in guppies ( Poecilia reticulata) in the Marianne watershed in northern Trinidad, and in the yellow pond lily (Nuphar lutea) in the Labe River watershed in the Czech Republic, respectively. All three watersheds are dendritic, with many headwater streams leading into successively larger main-stem reaches. Both of the fish species typically migrate asymmetrically downstream; additionally, their watersheds are fragmented by weirs (Rye) or waterfalls (Marianne), which cause much greater asymmetry in movement at specific locations. The yellow pond lily disperses its gametes primarily through hydrochory, which, potential animal vectors notwithstanding, is effectively unidirectional. As in the models we have presented, allelic richness generally increased from the headwaters to the main stem in all three systems, but more importantly, the increase in genetic variation was generally larger after the confluences of headwater below weirs or waterfalls rather than at unfragmented confluences (Crispo et al. 2006; Hanfling and Weetman 2006; Fer and Hroudova 2008; fig. 6). In addition, linear series of fragmented reaches generally did not have clines in allelic richness. A similar but less developed pattern has been reported in rainbow trout (Oncorhynchus mykiss) in the Russian River (central California; Deiner et al. 2007), in coastal cutthroat trout (Oncorhynchus clarki clarki) in Camp Creek (western Oregon; Wofford et al. 2005), and in white-spotted charr (Salvelinus leucomaenis) in the Ken-ichi River in Japan (Yamamoto et al. 2004). Barson et al. (2009) also studied guppy population genetics in the Marianne River and five surrounding watersheds in northern Trinidad and found that, at a coarse scale, genetic diversity was much higher in downstream populations in these highly dendritic watersheds. In contrast, these patterns were not found in the Scheldt River in Belgium, where threespine stickleback (Gasterosteus aculeatus) migration across weirs is downstream biased (Raeymaeker et al. 2008). However, this watershed is significantly larger ( $>10$ times) than the Rye or Marianne, potentially allowing other ecological interactions or unsampled populations to obscure the pattern. Finally, a recent study demonstrated that the number of upstream lakes is positively correlated with downstream zooplankton haplotype diversity in a series of lakes in Killarney Provincial Park, Ontario, Canada (Derry et al. 2009). Unfortunately, no studies of multiple intensively sampled watersheds that were of similar size but that varied in the degree of dendricity are available. We therefore cannot evaluate our more important finding that levels of genetic variation will generally be higher in dendritic systems.

Clearly, studies designed specifically to test for the joint effects of migration rate asymmetry and landscape dendricity would be very useful. To this end, several different 

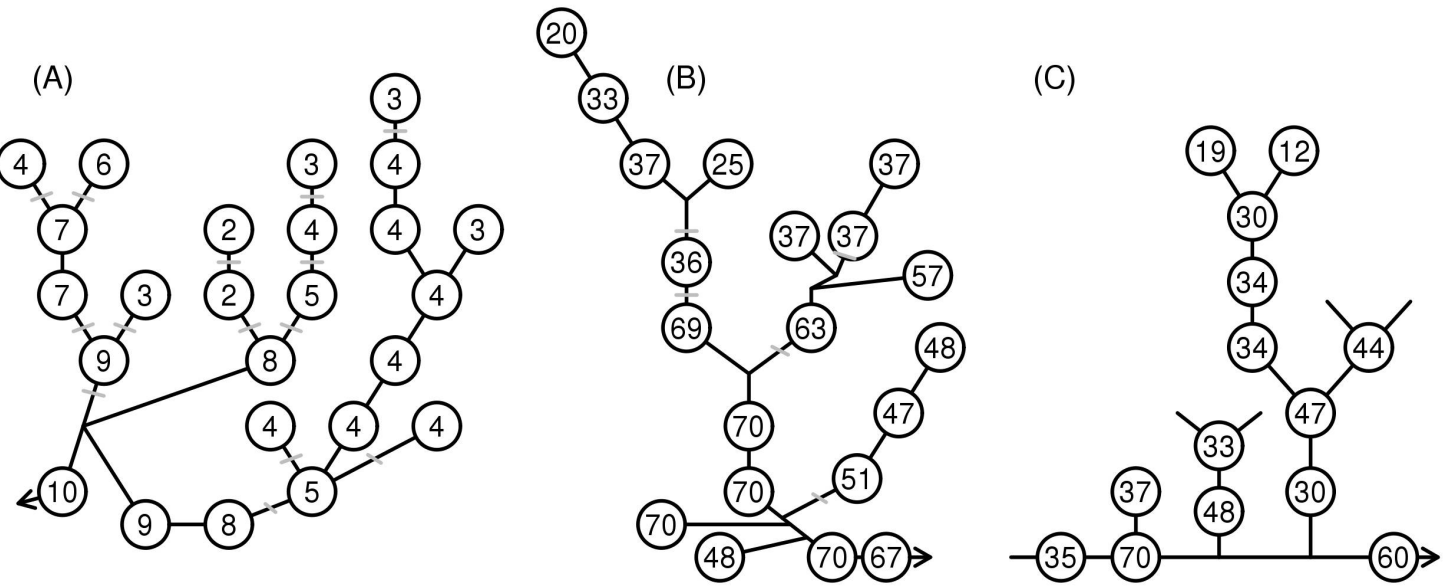

Figure 6: Allelic richness at sampling locations along rivers for two fishes, river sculpin Cottus gobio in England (A) and guppies Poecilia reticulata in Trinidad (B), and one plant, yellow water lilies Nuphar lutea in the Czech Republic (C). Note in some cases that the allelic richness is rounded to the nearest integer and that gray cross-hatching over the river indicates both artificial and natural barriers. In all of the examples, the reported heterozygosity was directly proportional to allelic richness, and for simplicity it was not reported. Modified from Hanfling and Weetman (2006; $A$ ), Crispo et al. (2006; B), and Fer and Hroudova (2008; C).

types of empirical systems may prove to be informative. Asymmetric migration in dendritic landscapes will be strongly mediated by the dispersal ability of the organism in question (Winemiller and Rose 1992; Bilton et al. 2001; de Kerckhove 2005), especially when dispersal is limited in the upstream direction (Shaw et al. 1994). Organisms with very limited swimming ability are clearly most likely to exhibit the patterns of increased levels of genetic variation in metapopulations that occur in our models (Schaefer 2001; Markwith and Scanlon 2007). However, a more interesting empirical problem is the extent to which the patterns that emerge from our models will occur in organisms that are capable of upstream dispersal. For example, swimming ability within fish species varies widely (Winemiller and Rose 1992), and fish exhibiting a subcarangiform (i.e., troutlike) swimming mode will ascend more instream velocity barriers than will similarly sized fish using an anguilliform (i.e., eel-like, highly undulating; Lindsay 1978) swimming mode (Katopodis and Gervais 1991).

Comparison of the effects of migration rate asymmetry in dendritic systems in the context of functional morphometry of fishes might provide a very promising avenue for empirical work. Previous research using this experimental design found predictable patterns of genetic variation of fish and invertebrate species in watersheds on the basis of their ability to ascend stream gradients and migrate over long distances (Monaghan et al. 2002; Whiteley et al. 2004; de Kerckhove 2005). Another promising approach would be the study of the same organism in different landscape arrangements (e.g., Castric et al. 2001).
Aquatic invertebrates may provide the most suitable study species because they are known to disperse over long distances by passive means (e.g., Bivalvia), but they also perform occasional upstream migrations by both animal vectors and their own movements. Organisms that are obligately aquatic and have little ability to swim for all but a very short period of their life cycles-for example, insects with aquatic larval stages - may provide interesting model systems as well. Clearly, dispersal of adults between headwaters could greatly disrupt the ability of headwater populations to act as reservoirs for unique alleles. However, this effect might be small in organisms with very short adult stages, for example, Ephemeroptera, but it could be very important in those with highly mobile and longerlasting adult stages, such as odonates (e.g., Chaput-Bardy et al. 2008).

\section{Model Considerations}

Extrapolation from the results of our limited simulations to expectations for patterns in genetic diversity in dendritic landscapes in nature will require consideration of a wide range of aspects of the natural history of dendritic landscapes and their inhabitants. For example, in metapopulations where headwaters are subjected to frequent cycles of extinction and recolonization (Fausch 1991), headwater populations may be less able to act as reservoirs of unique alleles for the downstream populations. Similarly, if some headwaters are more stable than others, or if they support larger populations than others, then they will likely contribute a larger proportion of migrants to the metapop- 
ulation. Such unequal contributions by individuals could lead to a breakdown of the tendency of our model systems to retard the erosion of genetic variation. However, it might be reasonable to expect highly developed dendritic systems (i.e., those with many different stream orders) to maintain high levels of diversity despite such demographic variation. The hierarchical nature of nodes in dendritic systems may allow for a filtering effect such that unequal contributions of headwater populations are mitigated by migration patterns of downstream populations (Campbell Grant et al. 2007). Thus, the effects of genetic swamping from one or a few emigrant-producing headwater populations may not necessarily strongly influence allele frequencies in other headwater populations. Similarly, populations in second-order or higher streams will be relatively genetically diverse in dendritic landscapes, and this may somewhat retard the homogenizing effect of extinction-recolonization effects when headwater populations are ephemeral.

Another simplifying assumption that we have made is that all demes are of equal size. We explore some effects of variation in local population size in the appendix. Local population sizes in nature are unlikely to conform strictly to this assumption. While the findings from our model will hold true when larger population sizes exist only in headwaters, a more interesting extension of our models occurs when larger downstream population sizes exist. This may occur in some watersheds, perhaps because stream size and, thus, habitat size may increase with stream order for some species. However, this will not be universally true, and patterns in population size may be the opposite for headwater-adapted species. An initial expectation might be that reduced drift in larger downstream populations supports the maintenance of genetic variation. However if individual migration probabilities are constant, a larger downstream population will contribute a larger number of upstream migrants and potentially swamp upstream populations, leading to a homogenization of upstream allele frequencies. These two mechanisms work in opposition to each other with respect to influences on genetic diversity. For a range of parameters, we have found that the second mechanism dominates over the first and reduces the relative effective size of the metapopulation. However, this reduction remains strongly influenced by the spatial arrangement of the landscape such that the patterns found in our models are still present between dendritic, uninodal, and linear landscapes (see appendix). Ultimately, however, the interplay between variation in population sizes and asymmetric migration in dendritic landscapes will vary among watersheds and species. So, although we have shown very strong effects of asymmetric migration in dendritic landscapes, further investigation of a wide range of demographic parameters is warranted.

\section{Conclusion}

In a recent review of the ecology of dendritic networks, Campbell Grant et al. (2007) commented on the lack of general theory relating to the ecological patterns and processes in natural systems with dendritic landscapes. In our review of available literature, we also found few theoretical treatments of asymmetric migration in non-lattice-type metapopulations (but see Lundy and Possingham 1998; Labonne et al. 2008) or empirical population genetic studies that offered appropriate systems. However, there is a great need to understand this interacting system, as asymmetry in migration is likely to be more common than perfectly symmetrical migration in metapopulations (Vuilleumier and Possingham 2006), and dendritic systems have already been demonstrated to act in very different ways from lattice-type networks (Fagan 2002; Campbell Grant et al. 2007; Rodriguez-Iturbe et al. 2009). Overall, our models demonstrate that the asymmetric conditions that typically lead to reduced diversity in lattice- and lineartype landscapes have the opposite effect-that is, to maintain genetic diversity-in dendritic networks.

\section{Acknowledgments}

We would like to acknowledge feedback and comments on the study and manuscript from P. Abrams, J. Biernaskie, B. Charlesworth, K. Johnson, M. Whitlock, A. Wilson, and two anonymous reviewers. The National Science and Engineering Research Council of Canada supported M.M. and D. de K. with graduate and postdoctoral awards.

\section{Literature Cited}

Barson, N. J., J. Cable, and C. Van Oosterhout. 2009. Population genetic analysis of microsatellite variation of guppies (Poecilia reticulata) in Trinidad and Tobago: evidence for a dynamic sourcesink metapopulation structure, founder events and population bottlenecks. Journal of Evolutionary Biology 22:485-497.

Benda, L., N. L. Poff, D. Miller, T. Dunne, G. Reeves, G. Pess, and M. Pollock. 2004. The network dynamics hypothesis: how channel networks structure riverine habitats. BioScience 54:413-427.

Bilton, D. T., J. R. Freeland, and B. Okamura. 2001. Dispersal in freshwater invertebrates. Annual Review of Ecology, Evolution, and Systematics 32:159-181.

Bishop, P. 1995. Drainage rearrangement by river capture, beheading and diversion. Processes in Physical Geography 19:449-473.

Blanchet, F. G., P. Legendre, and D. Borcard. 2008. Modelling directional spatial processes in ecological data. Ecological Modelling 215:325-336

Campbell Grant, E. H., W. H. Lowe, and W. F. Fagan. 2007. Living in the branches: population dynamics and ecological processes in dendritic networks. Ecology Letters 10:165-175.

Castric, V., F. Bonney, and L. Bernatchez. 2001. Landscape structure and hierarchical genetic diversity in the brook charr, Salvelinus fontinalis. Evolution 55:1016-1028.

Chaput-Bardy, A., C. Lemaire, C. Picard, and J. Secondi. 2008. In- 
stream and overland dispersal across a river network influences gene flow in a freshwater insect, Calopteryx splendens. Molecular Ecology 17:3496-3505.

Charlesworth, B., D. Charlesworth, and N. H. Barton. 2003. The effects of genetic and geographic structure on neutral variation. Annual Review of Ecology, Evolution, and Systematics 34:99-125. Chen, Y.-Y., W. Yang, W. Li, Z.-Z. Li, and H.-W. Huang. 2009. High allozyme diversity and unidirectional linear migration patterns within a population of tetraploid Isoletes sinensis, a rare and endangered pteridophyte. Aquatic Biology 90:52-58.

Crispo, E., P. Bentzen, D. N. Reznick, M. T. Kinnison, and A. P. Hendry. 2006. The relative influence of natural selection and geography on gene flow in guppies. Molecular Ecology 15:49-62.

Cuddington, K., and P. Yodzis. 2002. Predator-prey dynamics and movement in fractal environments. American Naturalist 160:119134.

DeAngelis, D. L., and W. M. Mooij. 2005. Individual-based modeling of ecological and evolutionary processes. Annual Review of Ecology, Evolution, and Systematics 36:147-168.

DeAngelis, D. L., M. Vos, W. M. Mooij, and P. A. Abrams. 2007. Feedback effects between the food chain and induced defense strategies. Pages 213-236 in N. Rooney, K. S. McCann, and D. L. G. Noakes, eds. From energetics to ecosystems: the dynamics and structure of ecological systems. Springer, Dordrecht.

Deiner, K., J. C. Garza, R. Coey, and D. J. Girman. 2007. Population structure and genetic diversity of trout (Oncorhynchus mykiss) above and below natural and man-made barriers in the Russian River, California. Conservation Genetics 8:437-454.

de Kerckhove, D. T. 2005. The influence of ecology and environment on the genetic population structure of three freshwater fish species. MS thesis. Department of Integrative Biology, University of Guelph, Guelph, Ontario.

Derry, A. M., S. E. Arnott, J. A. Shead, P. D. N. Hebert, and P. T. Boag. 2009. Ecological linkages between community and genetic diversity in zooplankton among boreal shield lakes. Ecology 90: 2275-2286.

Donnelly, P., and S. Tavaré. 1995. Coalescents and genealogical structure under neutrality. Annual Review of Genetics 29:401-421.

Ewens, W. J. 1982. On the concept of effective population size. Theoretical Population Biology 21:373-378.

Fagan, W. F. 2002. Connectivity, fragmentation, and extinction risk in dendritic metapopulations. Ecology 83:3243-3249.

Fausch, K. D. 1991. Disturbance and fish communities in intermittent tributaries of a western Great Plains river. Copeia 3:659-674.

Fer, T., and Z. Hroudova. 2008. Detecting dispersal of Nuphar lutea in river corridors using microsatellite markers. Freshwater Biology 53:1409-1422.

Finn, D. S., D. M. Theobald, W. C. Black IV, and N. L. Poff. 2006. Spatial population genetic structure and limited dispersal in a Rocky Mountain alpine stream insect. Molecular Ecology 15:35533566.

Fong, D. W., and D. C. Culver. 1994. Fine-scale biogeography differences in the crustacean fauna of a cave system in West Virginia, USA. Hydrobiologia 287:29-37.

Fraser, D. J., C. Lippé, and L. Bernatchez. 2004. Consequences of unequal population size, asymmetric gene flow and sex-biased dispersal on population structure in brook charr (Salvelinus fontinalis). Molecular Ecology 13:67-80.

Fulton, R. J. 1989. Quaternary geology of Canada and Greenland. Geological Survey of Canada, Ottawa.
Hamilton, M. 2009. Population genetics. Blackwell-Wiley, New York. Hanfling, B., and D. Weetman. 2006. Concordant genetic estimators of migration reveal anthropogenetically enhanced source-sink population structure in the river sculpin, Cottus gobio. Genetics 173: 1487-1501.

Hanski, I. 1999. Metapopulation ecology. Oxford Series on Ecology and Evolution. Oxford University Press, New York.

Haponski, A. E., T. A. Marth, and C. A. Stepien. 2007. Genetic divergence across a low-head dam: a preliminary analysis using logperch and greenside darters. Journal of Great Lakes Research $33: 117-126$.

Heggenes, J., and K. H. Red. 2006. Do dams increase genetic diversity in brown trout (Salmo trutta)? microgeographic differentiation in a fragmented river. Ecology of Freshwater Fish 15:366-375.

Hendry, A. P., and E. B. Taylor. 2004. How much of the variation in adaptive divergence can be explained by gene flow? an evaluation using lake-stream stickleback pairs. Evolution 58:2319-2331.

Katopodis, C., and R. Gervais. 1991. Icthyomechanics, working document. Fisheries and Oceans Canada, Freshwater Institute, Winnipeg, Manitoba.

Kimura, M., and G. H. Weiss. 1964. The stepping stone model of population structure and the decrease of genetic correlation with distance. Genetics 49:561-576.

Kirkpatrick, M., and N. H. Barton. 1997. Evolution of a species' range. American Naturalist 150:1-23.

Labonne, J., V. Ravigné, B. Parisi, and C. Gaucherel. 2008. Linking dendritic network structures to population demographics: the downside of connectivity. Oikos 117:1479-1490.

Lindsay, L. L. 1978. Form, function and locomotory habits in fish. Pages 1-100 in W. S. Hoar and D. J. Randall, eds. Fish physiology: locomotion. Vol. 17. Academic Press, New York.

Lowe, W. H. 2002. Landscape-scale spatial population dynamics in human-impacted stream systems. Environmental Management 30: 225-233.

Lowe, W. H., and D. T. Bolger. 2002. Local and landscape-scale predictors of salamander abundance in New Hampshire headwater streams. Conservation Biology 16:183-193.

Lundy, I. J., and H. P. Possingham. 1998. Fixation probability of an allele in a subdivided population with asymmetric migration. Genetical Research Cambridge 71:237-245.

MacArthur, R. H., and E. O. Wilson. 1967. The theory of island biogeography. Princeton University Press, Princeton, NJ.

Mandrak, N. E., and E. J. Crossman. 1992. Postglacial dispersal of freshwater fishes into Ontario. Canadian Journal of Zoology 70: 2247-2259.

Margan, S. H., R. K. Nurthen, M. E. Montgomery, L. M. Woodworth, E. H. Lowe, D. A. Briscoe, and R. Frankham. 1998. Single large or several small? population fragmentation in the captive management of endangered species. Zoo Biology 17:467-480.

Markwith, S. H., and M. J. Scanlon. 2007. Multiscale analysis of Hymenocallis coronaria (Amaryllidaceae) genetic diversity, genetic structure, and gene movement under the influence of unidirectional stream flow. American Journal of Botany 94:151-160.

Maruyama, T. 1969. Genetic correlation in the stepping stone model with non-symmetrical migration rates. Journal of Applied Probability 6:463-477.

- 1970. Effective number of alleles in a subdivided population. Theoretical Population Biology 1:273-306.

May, R. M. 2006. Network structure and the biology of populations. Trends in Ecology \& Evolution 21:394-399. 
Monaghan, M. T., P. Spaak, C. T. Robinson, and J. V. Ward. 2002. Population genetic structure of 3 alpine stream insects: influences of gene flow, demographics, and habitat fragmentation. Journal of the North American Benthological Society 21:114-131.

Muneepeerakul, R., E. Bertuzzo, H. J. Lynch, W. F. Fagan, A. Rinaldo, and I. Rodriguez-Iturbe. 2008. Neutral metacommunity models predict fish diversity patterns in Mississippi-Missouri basin. Nature 453:220-223.

Northcote, T. G. 1992. Migration and residency in stream salmonids: some ecological considerations and evolutionary consequences. Nordic Journal of Freshwater Research 67:5-17.

Palstra, F. P., and D. E. Ruzzante. 2008. Genetic estimates of contemporary effective population size: what can they tell us about the importance of genetic stochasticity for wild population persistence? Molecular Ecology 17:3428-3447.

Pulliam, H. R. 1988. Sources, sinks, and population regulation. American Naturalist 132:652-661.

Raeymaeker, J. A. M., G. E. Maes, S. Geldof, I. Hontis, N. Nackaerts, and F. A. M. Volckaert. 2008. Modeling genetic connectivity in sticklebacks as a guideline for river restoration. Evolutionary Applications 1:475-488.

Reid, S. M., C. C. Wilson, N. E. Mandrak, and L. M. Carl. 2008. Population structure and genetic diversity of black redhorse (Moxostoma duquesnei) in a highly fragmented watershed. Conservation Genetics 9:531-546.

Rodriguez-Iturbe, I., R. Muneepeerakul, E. Bertuzzo, S. A. Levin, and A. Rinaldo. 2009. River networks as ecological corridors: a complex systems perspective for integrating hydrologic, geomorphologic, and ecological dynamics. Water Resources Research 45:W01413.

Rosenberg, N. A., and M. Nordborg. 2002. Genealogical trees, coalescent theory and the analysis of genetic polymorphisms. Nature Reviews Genetics 3:380-390.

Saillant, E., J. C. Patton, K. E. Ross, and J. R. Gold. 2004. Conservation genetics and demographic history of the endangered Cape Fear shiner (Notropis mekistocholas). Molecular Ecology 13:29472958.

Schaefer, J. 2001. Riffles as barriers to interpool movement by three cyprinids (Notropis boops, Campostoma anomalum and Cyprinella venusta). Freshwater Biology 46:379-388.
Shaw, P. W., G. R. Carvalho, A. E. Magurran, and B. H. Seghers. 1994. Factors affecting the distribution of genetic variability in the guppy, Poecilia reticulata. Journal of Fish Biology 45:875-888.

Toju, H. 2008. Fine-scale local adaptation of weevil mouthpart length and camellia pericarp thickness: altitudinal gradient of a putative arms race. Evolution 65:1086-1102.

Velland, M. 2005. Species diversity and genetic diversity: parallel processes and correlated patterns. American Naturalist 166:199_ 215

Vuilleumier, S., and H. P. Possingham. 2006. Does colonization asymmetry matter in metapopulations? Proceedings of the Royal Society B: Biological Sciences 273:1637-1642.

Whiteley, A. R., P. Spruell, and F. W. Allendorf. 2004. Ecological and life history characteristics predict population genetic divergence of two salmonids in the same landscape. Molecular Ecology 13: 3675-3688.

Whitlock, M. C., and N. H. Barton. 1997. The effective size of a subdivided population. Genetics 146:427-441.

Wilson, C. C., and P. D. N. Hebert. 1998. Phylogeography and postglacial dispersal of lake trout (Salvelinus namaycush) in North America. Canadian Journal of Fisheries and Aquatic Sciences 55: 1010-1024.

Winemiller, K. O., and K. A. Rose. 1992. Patterns of life-history diversification in North American fishes: implications for population regulation. Canadian Journal of Fisheries and Aquatic Sciences 49:2196-2218.

Wofford, J. E. B., R. E. Gresswell, and M. A. Banks. 2005. Influence of barriers to movement on within-watershed genetic variation of coastal cutthroat trout. Ecological Applications 15:628-637.

Wright, S. 1931. Evolution in Mendelian population. Genetics 28 : 97-159.

Yamamoto, S., K. Morita, I. Koizumi, and K. Maekawa. 2004. Genetic differentiation of white-spotted charr (Salvelinus leucomaenis) populations after habitat fragmentation: spatial-temporal changes in gene frequencies. Conservation Genetics 5:529-538.

Associate Editor: Yannis Michalakis Editor: Donald L. DeAngelis 